\title{
Analyse Des Effets De L'adoption Des Variétés Améliorées De Maïs Sur La Sécurité Alimentaire Du Ménage De L’adoptant Au Nord-Bénin
}

\section{Yessifou Ayédesso Joski}

Université de Parakou. Département de l’Economie et Sociologie rurale. Laboratoire d'Analyse et de Recherche sur les Dynamiques Economiques et Sociales (LARDES),

Université de Parakou. Géographie économique. Laboratoire d'Analyse

Régionale et d'Expertise Sociale (LARES)

\section{Afouda Alix Servais}

Université de Parakou. Géographie économique. Laboratoire d'Analyse Régionale et d'Expertise Sociale (LARES)

Yabi Afouda Jacob

Université de Parakou. Département de l'Economie et Sociologie rurale. Laboratoire d'Analyse et de Recherche sur les Dynamiques Economiques et Sociales (LARDES)

Doi:10.19044/esj.2021.v17n29p298

Submitted: 22 June 2021

Accepted: 03 August 2021

Published: 31 August 2021
Copyright 2021 Author(s)

Under Creative Commons BY-NC-ND 4.0 OPEN ACCESS

Cite As:

Yessifou A.J.., Afouda A.S. \& Yabi A.J. (2021). Analyse Des Effets De L'adoption Des Variétés Améliorées De Maïs Sur La Sécurité Alimentaire Du Ménage De L'adoptant Au Nord-Bénin. European Scientific Journal, ESJ, 17(29), 298.

https://doi.org/10.19044/esj.2021.v17n29p298

\section{Résumé}

Pour analyser les effets de l'adoption des variétés améliorées de maïs (VAM) sur la sécurité alimentaire du ménage de l'adoptant, une étude a été réalisée auprès de 320 producteurs au Nord-Bénin plus précisément dans les Communes de Malanville, Banikoara, Kalalé et Djougou. Pour y parvenir les indicateurs de sécurité alimentaire tels que l'indice domestique de faim (HHS); les scores de consommation et de diversité alimentaire (SCAM et SDAM) et la part des dépenses alimentaires (PDA) des ménages ont été estimés et modélisés. Le test de ANOVA et le modèle de régression linéaire ont été respectivement utilisés pour la comparaison des moyennes et la modélisation des indicateurs. L'adoption des VAM réduit l'insécurité 
alimentaire du ménage parce que les résultats obtenus ont montré d'une part que les ménages adoptant les VAM ont des HHS, SCAM et SDAM plus élevés mais des PDA plus faibles que ceux des ménages non adoptants. Et d'autre part que ces indicateurs varient selon les caractéristiques socioéconomique et démographique du producteur. Alors pour que la culture du maïs renforce la sécurité alimentaire du ménage producteur, les gouvernants, projets et programmes doivent promouvoir davantage les variétés améliorées de maïs.

Mots clés : Adoption, VAM, Indicateurs, Sécurité Alimentaire, Nord-Benin

\section{Analysis of the effects of the adoption of improved varieties of maize on the food security of the producer's household in North Benin}

\section{Yessifou Ayédesso Joski}

Université de Parakou. Département de l’Economie et Sociologie rurale. Laboratoire d'Analyse et de Recherche sur les Dynamiques Economiques et Sociales (LARDES),

Université de Parakou. Géographie économique. Laboratoire d'Analyse Régionale et d'Expertise Sociale (LARES)

\section{Afouda Alix Servais}

Université de Parakou. Géographie économique. Laboratoire d'Analyse Régionale et d'Expertise Sociale (LARES)

\section{Yabi Afouda Jacob}

Université de Parakou. Département de l'Economie et Sociologie rurale. Laboratoire d'Analyse et de Recherche sur les Dynamiques Economiques et Sociales (LARDES)

\section{Abstract}

To assess the effects of the adoption of improved varieties of maize (VAM) on the food security of the adopting household, a study was carried out among 320 producers in North Benin, more specifically in the Communes of Malanville, Banikoara, Kalalé and Djougou. To achieve this, food security indicators such as the Household Hunger Scale (HHS); the food consumption and diversity scores (SCAM and SDAM) and the share of household food expenditure (PDA) were estimated and modeled. The ANOVA test and the linear regression model were respectively used for comparison of means and modeling of indicators. The adoption of VAM reduces household food insecurity because the results obtained have shown on the one hand that households adopting VAM have higher HHS, SCAM and SDAM but lower 
PDAs than those of non-adopting households. On the other hand, these indicators vary according to the socioeconomic and demographic characteristics of the producer. So that the cultivation of maize strengthens the food security of the producer household, governments, projects and programs must further promote improved varieties of maize.

Keywords: Adoption, VAM, Indicators, Food Security, North Benin

\section{Introduction}

La production agricole et les revenus qu'elle génère doivent normalement permettre aux ménages agricoles de satisfaire leurs besoins fondamentaux. Mais l'expérience alimentaire du ménage agricole consiste souvent aux accès limités en nourritures avec une insuffisance en qualités nutritives(Yaï et al., 2020). En effet l'agriculture dans certains pays africains est caractérisée par une baisse de la productivité (Dury et al., 2017b; Jayne et al., 2010). Cette baisse de la productivité devient progressive surtout dans la décennie de 2010-2020 (Bucekuderhwa et al., 2013). Conséquemment, les ménages souffrant d'insécurité alimentaire sont accrus et la grande partie des populations agricoles, orientés vers le champ, vivent sans satisfaire leurs besoins alimentaires (Dury et al., 2012).

En effet la problématique de la sécurité alimentaire et nutritionnelle est l'un des enjeux majeurs au Bénin. Sous-tendue par des causes multifactorielles et interdépendantes, la situation alimentaire et nutritionnelle présente des disparités insidieuses entre les zones rurales et urbaines. Pendant longtemps, les politiques et les interventions en faveur de l'amélioration de la situation alimentaire des populations se sont focalisées sur les zones rurales considérées comme des zones « à priori vulnérables » (EPA, 2019).

Pour réduire l'insécurité alimentaire au Bénin et en particulier dans les zones cotonnières, il convient d'investir aussi dans les produits céréaliers et animaux(Gérard et al., 2012; Affholder et al., 2013), fortement concurrencés par la production du coton. Le rapport de la division statistique de la FAO en 2015 a révélé que parmi les céréales, la culture du maïs est en tête en Afrique avec une superficie emblavée d'environ 34075972 ha et une production annuelle de 70076591 tonnes. De ce fait, 208 millions de personnes en Afrique Sub-Saharienne dépendent du maïs comme source de sécurité alimentaire et de bien-être économique (Macauley et al., 2015).

Au Bénin le maïs (Zea mays L.), largement cultivé sur toute l'étendue du territoire national, occupe près de $70 \%$ des superficies céréalières emblavées. Il est à ce jour la céréale la plus consommée loin devant le riz et le sorgho (Pomalegni et al., 2019). Malgré tous les efforts consentis, l’offre de maïs est loin de satisfaire la demande sans cesse croissante et les rendements sont de plus en plus faibles du fait des fréquentes perturbations climatiques. Il 
est clair que la contrainte principale à laquelle se trouve confrontée la filière est sa faible productivité (CORAF /WECARD, 2018). Pour remédier à cette baisse de la productivité afin d'améliorer le revenu et la sécurité alimentaire du ménage producteur, les instituts de recherche ont mis au point des semences de variétés améliorées. Au niveau de la vulgarisation agricole, on compte treize (13) variétés améliorées à haut rendement de maïs (Yallou et al., 2010) et selon la présente étude, seules les semences de cinq (5) variétés telles que DMRESRW, FAABA.Obatampa, EVDT97STRW, TZPBSRW et 2000Syn.EEW sont effectivement utilisées par les producteurs de la zone de recherche. Après quelques décennies de vulgarisations, il s'avère nécessaire d'analyser les effets de leur adoption sur la sécurité alimentaire des ménages des producteurs adoptants.

Plusieurs études ont abordé les questions de la sécurité alimentaire et des semences améliorées au Benin. Yaï et al. (2020), dans leur étude sur la productivité agricole et sécurité alimentaire des ménages agricoles du Bénin, ont montré que l'accroissement de la productivité agricole serait efficace pour la réalisation de la sécurité alimentaire des ménages agricoles du Bénin. Saliga et al. (2021) dans leurs travaux sur la sécurité alimentaire des ménages agricoles dans le département du Borgou en République du Bénin ont trouvé qu'il urge de mettre en place des mécanismes qui incitent les ménages agricoles vulnérables à adopter des stratégies préventives et la pluriactivité. Quant aux travaux de Grethe et al. (2020), sur l'analyse ex-ante du "Plan National d'Investissements Agricoles et de Sécurité Alimentaire et Nutritionnelle“ du Benin ils, ont abouti à la conclusion que les ménages les plus riches ont une situation alimentaire plus améliorée que celle des ménages pauvres et que les ménages ruraux les plus pauvres subissent même une perte de revenu. Adekambi et al. (2020), dans leur recherche sur l'effet combiné de l'adoption de légumineuses comme précédent cultural et des variétés améliorées de maïs sur le bien-être des producteurs au Nord du Bénin, ont montré que l'adoption combinée des deux technologies a permis d'améliorer la sécurité alimentaire des ménages. Dans le même temps il a été démontré par Akpo (2020) que l'adoption des variétés améliorées de semences présente des effets nettement positifs comparativement à l'utilisation des semences locales.

Ces différentes études ont abordé la problématique de la sécurité alimentaire avec des approches différentes sans mettre un accent particulier sur l'influence que peut avoir la culture des variétés améliorées de maïs sur la sécurité alimentaire. Et comme l'ont notifié Diouf Sarr et al. (2018) pour la région de Fatick, peu d'études ont évalué les effets de l'adoption des variétés améliorées de maïs sur la sécurité alimentaire des ménages au Bénin et plus précisément dans la région septentrionale où la production agricole est importante. 
Afin de combler cette insuffisance et d'apprécier au mieux l'influence des VAM sur la sécurité alimentaire, notre étude vient se compléter à celles existantes tout en exploitant une approche qui puisse montrer clairement les aspects de la situation alimentaire du ménage producteur.

Car la connaissance de l'état de sécurité alimentaire des producteurs de maïs peut aider les exploitations agricoles à assurer la disponibilité des aliments de base dans les ménages tout au long de l'année à travers le renforcement de leur capacité de production, l'adoption des variétés de semences requises et d'intrants de meilleure qualité. Ainsi, la question pertinente qui se pose est la suivante : Quels sont les effets de l'adoption des variétés améliorées de maïs sur la sécurité alimentaire du ménage adoptant au Nord-Bénin?

\section{Matériel et Méthodes \\ Choix des sites d'étude}

La zone Nord du Bénin selon les prévisions serait très vulnérable aux effets néfastes du changement climatique. En effet, une réduction de 13 à 15\% de précipitation à l'horizon 2100 est prévisible, ce qui aurait d'impacts négatifs très importants sur les cultures installées dans les différentes zones agro écologiques de cette région (Yegbemey et al., 2014). En termes de potentialité agricole, d'importantes superficies sont consacrées à la production céréalière en générale et celle du maïs en particulier (Kayodé, 2018). Cette étude s'est déroulée dans quatre zones agro écologiques caractéristiques de la région Nord du pays. Sur la base de la revue documentaire, une (01) Commune a été choisie par zone agro écologique. Il s'agit de Malanville (Zone 1), de Banikoara (Zone 2), de Kalalé (Zone 3) et de Djougou (Zone 4) (figure 1). Les différentes Communes ont été choisies non seulement parce qu'elles sont caractéristiques de ces différentes zones agro écologiques au Nord-Bénin, mais aussi parce qu'elles sont réputées productrices de maïs et représentent des zones où les risques liés à la sécheresse sont très élevés (MEPN, 2008). Elles ont d'ailleurs bénéficié du soutien du Programme d'Action National aux fins de l'Adaptation aux changements climatiques de 2007 à 2013, et du projet d'appui à la production vivrière et de renforcement de la résilience depuis 2015. Dans chaque Commune, le choix des villages s'est fait après une phase exploratoire. Cette phase a été caractérisée par des entretiens semi-structurés avec les agents d'ATDA (Agence Territoriale du Développement Agricole) qui est abouti à un classement des villages, les plus vulnérables aux moins vulnérables, en tenant compte de leurs productivités en maïsiculture. A l'issu de ce classement, deux villages ont été retenus par Commune échantillonnée. Il s'agit de Partago et Dabogou pour la Commune de Djougou, Kalalé centre et de Dangazi pour la Commune de Kalalé, de Simpérou et Poto pour la 
Commune de Banikoara et puis de Kassa et Madécalli pour la Commune de Malanville (Figure 1).
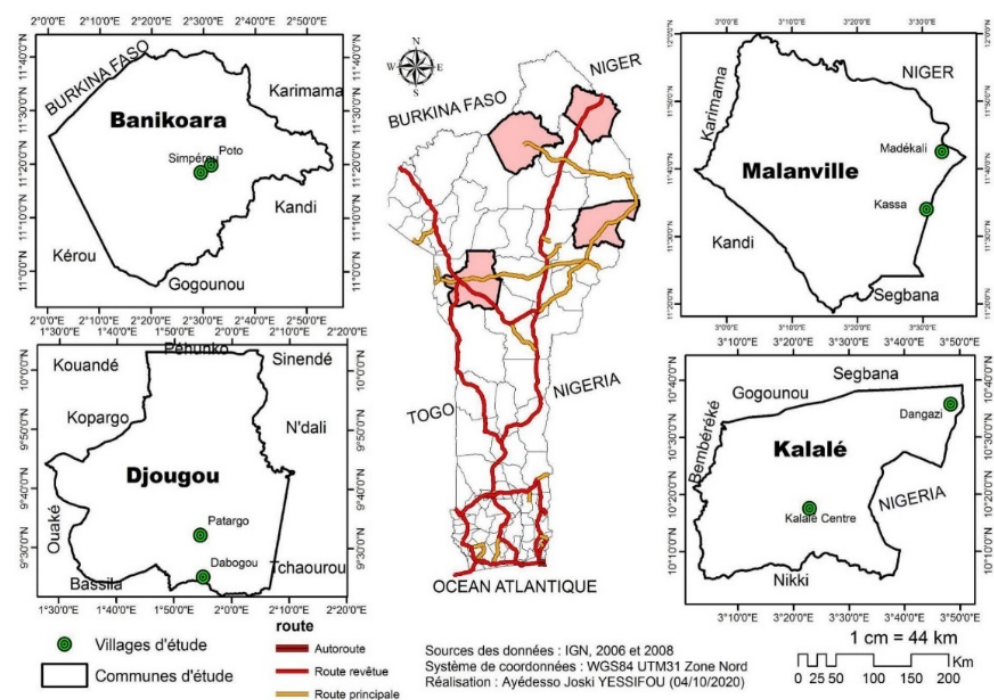

Figure 1. Situation géographique des sites d'étude

Source : Auteurs à partir des données de l’IGN 2006

\section{Échantillonnage}

L’unité d'échantillonnage dans cette étude est le producteur de maïs. La taille minimale d'échantillon a été déterminée à l'aide de la formule de Rea (1997). Cette formule s'écrit comme suit :

$$
x=\frac{\mathrm{t}_{\mathrm{p}}^{2}(1-p) \times N \times \mathrm{p}}{\mathrm{t}_{\mathrm{p}}^{2} \times \mathrm{p}(1-\mathrm{p})+(\mathrm{N}-1) \mathrm{y}^{2}}
$$

Où

\begin{tabular}{|l|l|}
\hline $\boldsymbol{x}$ & : la taille de l'échantillon \\
\hline $\mathbf{N}$ & : nombre de ménages agricoles pour chaque Commune \\
\hline $\mathbf{p}$ & $\begin{array}{l}\text { : la proportion de ménages agricoles produisant le maïs et pouvant accéder aux } \\
\text { semences améliorées dans la zone de recherche (d'après une phase exploratoire initiée } \\
\text { on constate que sur } 100 \text { producteurs de maïs environ } 75 \text { ont accès aux variétés } \\
\text { améliorées soit p=0,75 dans l'ensemble de la zone de recherche) }\end{array}$ \\
\hline $\mathbf{t}_{\mathbf{p}}$ & $\begin{array}{l}\text { : Valeur associée à un intervalle de confiance d'échantillonnage donné. Pour cette } \\
\text { étude, tp est égale à 1,96 pour un intervalle de confiance de 95\%. C'est-à-dire la } \\
\text { probabilité que l'échantillon de personnes interrogées ait une influence sur les résultats } \\
\text { de l'enquête est de 95\%. En plus l'intervalle de confiance couramment utilisé est de } \\
95 \% .\end{array}$ \\
\hline $\mathbf{y}$ & $\begin{array}{l}\text { : la marge d'erreur d'échantillonnage (5\%). En effet, une marge d'erreur de 5\% est } \\
\text { communément considérée comme suffisante. }\end{array}$ \\
\hline
\end{tabular}

Sur la base de la précédente formule, des données provenant du tableau 1 et en prenant une marge d'erreur de $5 \%$ avec un intervalle de confiance de 95\%, la taille minimale de notre échantillon est de 270,43 ménages 
producteurs du maïs. L'échantillonnage a été porté à 320 et pour s'assurer d'atteindre la taille définie pour l'échantillon minimal les nombres de personnes à enquêter par village ont été proportionnellement augmentés. En effet, plus la taille de l'échantillon augmente plus les paramètres mesurés sont fiables (Sossou, 2015). Le tableau 1 renseigne le nombre de producteurs de maïs enquêté par Commune suivant les villages.

Tableau 1. Taille d'échantillonnage par village sélectionné

\begin{tabular}{|c|c|c|c|c|c|c|}
\hline $\begin{array}{c}\text { Zones agro } \\
\text { écologiques }\end{array}$ & Communes & Arrondissement & $\begin{array}{c}\text { Nombre de } \\
\text { ménages } \\
\text { producteurs } \\
\text { de maïs }\end{array}$ & $\begin{array}{l}\text { Villages } \\
\text { retenus }\end{array}$ & Effectifs & Pourcentage \\
\hline \multirow[t]{3}{*}{ ZAE I } & \multirow[t]{2}{*}{ Malanville } & \multirow[t]{2}{*}{ Madécali } & \multirow[t]{2}{*}{938} & Kassa & 23 & $7 \%$ \\
\hline & & & & Madécalli & 46 & $14 \%$ \\
\hline & & & & Sous- total & 69 & $21 \%$ \\
\hline \multirow[t]{3}{*}{ ZAE II } & \multirow[t]{2}{*}{ Banikoara } & \multirow[t]{2}{*}{ Somperekou } & \multirow[t]{2}{*}{1096} & Poto & 56 & $18 \%$ \\
\hline & & & & Simpérou & 25 & $8 \%$ \\
\hline & & & & Sous- total & 81 & $26 \%$ \\
\hline \multirow[t]{3}{*}{ ZAE III } & \multirow[t]{2}{*}{ Kalalé } & \multirow[t]{2}{*}{ Kalalé centre } & \multirow[t]{2}{*}{1318} & Dangazi & 49 & $15 \%$ \\
\hline & & & & Kalalé centre & 45 & $14 \%$ \\
\hline & & & & Sous- total & 94 & $29 \%$ \\
\hline \multirow[t]{3}{*}{ ZAE IV } & \multirow[t]{2}{*}{ Djougou } & \multirow[t]{2}{*}{ Partago } & \multirow[t]{2}{*}{1038} & Dabogoun & 19 & $6 \%$ \\
\hline & & & & Partago & 57 & $18 \%$ \\
\hline & & & & Sous- total & 76 & $24 \%$ \\
\hline \multicolumn{3}{|c|}{ Total } & 4390 & & 320 & $100 \%$ \\
\hline
\end{tabular}

Source: Données de RGPH4 (INSAE, 2016) et Données d'enquête, 2020

\section{Approche d'évaluation de la sécurité alimentaire à l'échelle d'un ménage}

Plusieurs méthodes permettent d'analyser la sécurité alimentaire dans un ménage. Selon une analyse comparative examinant quatre types de mesure proxy de 1 'accès alimentaire; à savoir les indicateurs de disponibilité calorique, de pauvreté monétaire, de diversité alimentaire et de mesure subjective (comme les échelles basées sur l'expérience), Headey et al. (2013) ont déterminé que les indicateurs de diversité alimentaire étaient les plus performants en termes de leur association aux mesures du statut socioéconomiques et au statut nutritionnel (malnutrition aigüe et chronique). Ces indicateurs étaient aussi plus sensibles à la variabilité temporelle et plus économique à administrer(Headey et al., 2013). Malgré ces avantages, l'interprétation de la diversité alimentaire comme mesure d'accès a été critiquée(Jones et al., 2014; Legwegoh et al., 2013; Vellema et al., 2014). Donc pour des raisons valables, «linsécurité alimentaire» n'a pas de métrique de référence acceptée par rapport à laquelle les indicateurs individuels peuvent être évalués, mais sans un, il est difficile de dire quel indicateur fonctionne «le mieux» pour identifier correctement et de manière fiable les ménages en 
insécurité alimentaire. L'implication est que l'utilisation de plus d'un indicateur est souhaitable, et les décideurs doivent être conscients des éléments de l'insécurité alimentaire que chaque indicateur représente (Maxwell et al., 2014). Pour analyser l'état de la sécurité alimentaire des ménages (Mulumeoderhwa et al., 2020) ont considéré deux indicateurs importants dont la part des dépenses alimentaires dans le budget des ménages et le score de consommation alimentaire. Pour la même cause Michikpe et al. (2017) ont pris pour outils HHS (Household Hunger Scale ou indice domestique de faim) et le SCAM (Score de Consommation Alimentaire du Ménage). Ce qui semble insuffisant car ces deux approches, même combinées, ne renseignent pas de façon claire sur la diversité des aliments consommés dans le ménage. Afin d'apprécier au mieux la sécurité alimentaire des ménages, le HHS (Household Hunger Scale ou l'indice domestique de faim) a été estimé pour surveiller la prévalence de la faim au fil du temps dans la zone recherche (Ballard et al., 2011) ou pour mesurer la sévérité de l'insécurité alimentaire (Michikpe et al., 2017). En suite le Score de Consommation Alimentaire (SCAM) et le Score de Diversité Alimentaire (SDAM) pouvant témoigner l'accessibilité, la disponibilité, la consommation et la diversité des aliments consommés dans le ménage seront évalués. En outre, le SCAM et le SDAM sont des scores très utilisés par le FAO; le PAM et l'USAID pour analyser la sécurité alimentaire (Ndiaye, 2014). Enfin pour chaque ménage la part des dépenses alimentaires ou la vulnérabilité économique (PDA) sera estimé car la structure des dépenses totales influe sur la consommation alimentaire (Bocoum, 2012). Ce dernier indicateur, très pertinent mais peu utilisé, est basé sur le postulat que plus la part du budget consacrée aux aliments a de l'importance au sein du budget d'un ménage (relativement aux autres biens /services consommés), plus le ménage est vulnérable économiquement. Et il intervient dans le calcul de l'indice de sécurité alimentaire (Paridaens et al., 2017).

\section{L'indice domestique de faim HHS :}

HHS ou indice domestique de faim (Maxwell et al., 1992) est un indicateur simple pour mesurer la faim dans les ménages en zones d'insécurité alimentaire. L'approche utilisée par le HHS est basée sur l'idée que l'expérience de la privation alimentaire dans les ménages provoque des réactions prévisibles qui peuvent être saisies grâce à une enquête et résumées dans un indice (Michikpe et al., 2017).

Selon Ballard et al. (2011) l'évaluation du HHS s'effectue en trois étapes à savoir :

Étape 1. La première étape consiste à recoder les réponses à chaque question sur la fréquence d'événement de trois catégories de fréquence (" rarement ", " parfois », " souvent ») en deux catégories de fréquence (« rarement ou parfois » et " souvent »). Pour éviter de perdre les données 
recueillies à l'origine, créer une nouvelle variable pour chaque question de fréquence de l'événement. N'écrasez pas les données originales. Ici, référence est faite aux nouvelles variables créées pour chaque question de fréquence d'événement comme NouvelleQ1, NouvelleQ2 et NouvelleQ3. Pour chacune des nouvelles variables créées, une réponse de fréquence de " rarement » (codée à l’origine comme « 1 ») est codée « 1 », une réponse de fréquence de " parfois » (codée à l'origine comme « 2 ») est codée « 1 » et une réponse de fréquence de « souvent » (codée à l'origine comme « 3 ») est codée « 2 ».

Étape 2. Ensuite, ajoutez un code de « 0 » pour les ménages qui ont répondu « Non » à chaque question d'événement correspondante. Une fois cette étape est terminée, tous les ménages doivent avoir une valeur de 0,1 ou 2 pour chacune des trois nouvelles variables créées, NouvelleQ1, NouvelleQ2 et NouvelleQ3.

Étape 3. Les valeurs de NouvelleQ1, NouvelleQ2 et NouvelleQ3 sont ensuite additionnées pour chaque ménage afin de calculer le score du HHS. Si la totalisation a été effectuée correctement, chaque ménage aura un score du HHS entre 0 et 6 . Ces valeurs sont ensuite utilisées pour générer les indicateurs du HHS (Tableau 4).

Le tableau 2 présente les trois questions Q1, Q2 et Q3 qui forment le HHS.

Tableau 2. Récapitulatif des trois questions du HHS

\begin{tabular}{|c|c|}
\hline Question du HHS & Sens voulu de la question du HHS \\
\hline $\begin{array}{c}\text { Qucun aliment } \\
\text { dans la maison }\end{array}$ & $\begin{array}{c}\text { Cette question porte sur une situation dans laquelle le ménage n'a } \\
\text { rien à manger à la maison, parce que la nourriture n'était pas } \\
\text { disponible pour les membres du ménage à travers les moyens } \\
\text { habituels d'approvisionnement. }\end{array}$ \\
\hline $\begin{array}{c}\text { Q2: Dormir affamé } \\
\text { parce qu'il n'y } \\
\text { avait pas assez de } \\
\text { nourriture }\end{array}$ & $\begin{array}{c}\text { Cette question demande si la personne interrogée ou les autres } \\
\text { membres du ménage ont eu faim au moment d'aller au lit parce } \\
\text { qu'ils n'avaient pas assez mangé dans la journée et le soir. }\end{array}$ \\
\hline $\begin{array}{c}\text { Q3: Passer un jour } \\
\text { et une nuit entiers } \\
\text { sans manger }\end{array}$ & $\begin{array}{c}\text { Cette question demande si l'un des membres du ménage n'a rien } \\
\text { mangé depuis son réveil ce matin jusqu'à son réveil le lendemain } \\
\text { matin parce qu'il n'y avait pas assez de nourriture. Une personne } \\
\text { qui choisit de ne pas manger pendant toute une journée pour des } \\
\text { raisons autres que le manque de nourriture. }\end{array}$ \\
\hline
\end{tabular}

Source : Ballard et al. (2011)

\section{Traduction Mathématique du HHS}

D'après la revue de littérature on peut retenir que l'indicateur catégorique du HHS est évalué à travers les réponses aux trois questions piliers du HHS. Soit Ri la valeur attribuée à la réponse de la question Qi.

$$
\boldsymbol{H} \boldsymbol{H} \boldsymbol{S}=\sum_{i=1}^{\mathbf{3}} \boldsymbol{R i} \leq \mathbf{6} \quad \operatorname{car}\{R i \in(0,1,2) \text { et } i \in(1,2,3)\}
$$


Interprétation du HHS: Les trois catégories de la faim dans les ménages sont présentées le tableau ci-dessous.

Tableau 3. L'indicateur catégorique du HHS

\begin{tabular}{|cc|c|}
\hline \multicolumn{2}{|c|}{ Valeurs Seuils du HHS } & Catégorie de faim \\
\hline $\mathrm{Si}$ & $0 \leq \mathrm{HHS} \leq 1$ & Peu ou pas de faim \\
\hline $\mathrm{Si}$ & $2 \leq \mathrm{HHS} \leq 3$ & Faim modérée dans le ménage \\
\hline $\mathrm{Si}$ & $4 \leq \mathrm{HHS} \leq 6$ & Faim sévère dans le ménage \\
\hline
\end{tabular}

Source: Ballard et al. (2011)

\section{Score de Diversité Alimentaire des Ménages (SDAM)}

Le SDAM est une mesure proxy de l'accès des ménages à une alimentation variée. Conçu pour sa facilité d'utilisation et largement promu par la FAO et l'USAID (Ndiaye, 2014), il est un indicateur de la couverture des besoins en micronutriments (Martin-Prével et al., 2015) qui tient compte du nombre de groupes alimentaires qui ont été consommés par les ménages au cours des dernières 24 heures et ce indépendamment de la valeur nutritionnelle de ces groupes alimentaires (Paridaens et al., 2017). Les groupes d'aliment utilisé dans le tableau 5.

Tableau 4. Groupes d'aliment entrant dans le calcul du SDAM

\begin{tabular}{|c|c|c|}
\hline & $\begin{array}{c}\text { Groupes d'aliments } \\
\text { pour SDAM }\end{array}$ & Aliments appartenant au groupe \\
\hline \multirow[t]{2}{*}{1} & \multirow{2}{*}{$\begin{array}{l}\text { Céréales et } \\
\text { tubercules }\end{array}$} & Riz, pâtes, pain/cake, sorgho, mais, mil \\
\hline & & Patates, manioc, patate douce, taro et/ou autres racines \\
\hline 2 & Légumineuses & Haricots, pois, arachides, lentilles, noix, soja et / ou autres noix \\
\hline \multirow[t]{3}{*}{3} & \multirow[t]{3}{*}{ Légumes } & Carotte, poivron rouge, courge, patate douce orange \\
\hline & & Epinards, brocolis, amarante et/ou autres feuilles vert foncé \\
\hline & & $\begin{array}{c}\text { Oignon, tomates, concombre, radis, haricots verts, petits pois, } \\
\text { salade, etc. }\end{array}$ \\
\hline \multirow[t]{2}{*}{4} & \multirow[t]{2}{*}{ Fruits } & Mangue, papaye, abricot, pèche \\
\hline & & banane, pomme, citron, clémentine \\
\hline \multirow[t]{2}{*}{5} & \multirow[t]{2}{*}{ Viandes et Poissons } & Chèvre, bœuf, poulet, porc \\
\hline & & $\begin{array}{c}\text { Poisson, thon en boite inclus, escargot, et / ou autres fruits de la } \\
\text { mer }\end{array}$ \\
\hline 6 & Lait & $\begin{array}{c}\text { Lait frais, yaourt, fromage, et autres produits laitiers (Exclure } \\
\text { Margarine / beurre ou les petites quantités de lait dans le } \\
\text { lait/café) }\end{array}$ \\
\hline 7 & Huile & Huile végétale, huile de palme, margarine, autres graisses/huiles \\
\hline
\end{tabular}

Source : Ndiaye (2014)

\section{Traduction mathématique du SDAM}

D’après Ndiaye (2014) pour calculer le SDAM:

a-) on crée pour chaque groupe alimentaire une nouvelle variable binomiale qui peut prendre 2 valeurs:

*1-Si oui: le ménage/individu a consommé un aliment de ce groupe *0-Sinon: le ménage/individu n’a pas consommé un aliment de ce groupe. 
b-) on additionne toutes les variables binomiales afin de créer un SDAM. La nouvelle variable aura une valeur comprise entre 0 et 7 (le nombre de groupe d'aliments collectés). Mathématiquement traduit on a:

$$
S D A M=\sum_{i=1}^{7} \alpha_{i}
$$

Où $\alpha_{i}$ est la variable binomiale créée pour chaque groupe alimentaire.

Interprétation du SDAM: La norme minimale du SDAM est fixée à ' 4 '”par le PNUD (2015). Les ménages ayant consommé au minimum quatre groupes alimentaires différents ont une diversité alimentaire qualifiée d'acceptable. Plus le nombre de groupes alimentaires consommés augmente, plus la consommation alimentaire s'améliore (Paridaens et al., 2017).

\section{Score de Consommation Alimentaire des Ménages (SCAM)}

Le SCAM est la somme des nombres de jours de consommation des groupes d'aliments multipliés par leur coefficient (Headey et al., 2013). C'est un score composite utilisé comme indicateur de substitution de la consommation alimentaire et donc de l'accès à l'alimentation. Il reflète la quantité (kcal) et qualité (nutriments - importance nutritionnelle) de l'alimentation. Basé sur un rappel de 7 jours précédant l'enquête sur les types/groupes d'aliments et la fréquence de consommation. Il est calculé en se basant sur la fréquence de consommation des différents groupes d'aliments consommés par un ménage. Il est principalement utilisé par le PAM (Programme Alimentaire Mondial) (Ndiaye, 2014). Les informations qualitatives recueillies sur les fréquences de consommation des différents groupes d'aliment (Tableau 6) ont servi à calculer le score de la consommation alimentaire.

Traduction mathématique du SCAM : Pour le calculer on fait la somme des produits de la pondération et du nombre de jours de consommation de chaque groupe d'aliment (Tableau 6). Ce qui se traduit mathématiquement par la formule suivante :

$$
S C A M=\sum_{i=1}^{n} \operatorname{POnGrAl}_{i} \times N C_{i}
$$

Où : PonGrAl ${ }_{i}$ est la pondération du groupe d'aliment ' ' $i$ ', et $N_{i}$ est le nombre de jours de consommation d'un aliment appartenant au groupe d'aliment " $i$ "'

Les groupes d'aliment et les pondérations correspondantes sont mentionnés dans le tableau suivant. 
Tableau 5. Groupes d'aliments et pondérations correspondantes permettant le calcul du SCAM

\begin{tabular}{|c|c|c|c|}
\hline & $\begin{array}{l}\text { Groupe d'aliments du } \\
\text { SCAM } \\
\end{array}$ & Aliments appartenant au groupe & $\begin{array}{l}\text { Pondé } \\
\text { rations }\end{array}$ \\
\hline 1 & Céréales et graines & Riz, pâtes, pain/cake, sorgho, maïs, mil & \multirow[t]{2}{*}{2} \\
\hline 2 & Racines et tubercules & $\begin{array}{c}\text { Patates, manioc, patate douce, taro et/ou autres } \\
\text { racines }\end{array}$ & \\
\hline 3 & Légumineuses & $\begin{array}{c}\text { Haricots, pois, arachides, lentilles, noix, soja et / ou } \\
\text { autres noix }\end{array}$ & 3 \\
\hline 4 & $\begin{array}{l}\text { Végétaux riches en } \\
\text { vitamine A }\end{array}$ & Carotte, poivron rouge, courge, patate douce orange & \multirow[t]{3}{*}{1} \\
\hline 5 & $\begin{array}{l}\text { Légumes verts foncés à } \\
\text { feuilles }\end{array}$ & $\begin{array}{l}\text { Epinards, brocolis, amarante et/ou autres feuilles vert } \\
\text { foncé }\end{array}$ & \\
\hline 6 & Autres légumes & $\begin{array}{c}\text { Oignon, tomates, concombre, radis, haricots verts, } \\
\text { petits pois, salade, etc. }\end{array}$ & \\
\hline 7 & $\begin{array}{c}\text { Fruits riches en vitamine } \\
\text { A }\end{array}$ & Mangue, papaye, abricot, pèche & \multirow[t]{2}{*}{1} \\
\hline 8 & Autres fruits & Banane, pomme, citron, clémentine & \\
\hline 9 & Viande & Chèvre, bœuf, poulet, porc & \multirow[t]{4}{*}{4} \\
\hline 10 & $\begin{array}{l}\text { Foie, reins, cœur et/ou } \\
\text { autres organes }\end{array}$ & & \\
\hline 11 & Poisson/Fruits de la mer & $\begin{array}{l}\text { Poisson, thon en boite inclus, escargot, et / ou autres } \\
\text { fruits de la mer }\end{array}$ & \\
\hline 12 & Eufs & & \\
\hline 13 & Laits et produits laitiers & $\begin{array}{c}\text { Lait frais, yaourt, fromage, et autres produits laitiers } \\
\text { (Exclure margarine / beurre ou les petites quantités } \\
\text { de lait dans le lait/café) }\end{array}$ & 4 \\
\hline 14 & Huile/graisse/beurre & $\begin{array}{c}\text { Huile végétale, huile de palme, margarine, autres } \\
\text { graisses/huiles }\end{array}$ & 0,5 \\
\hline 15 & Sucre ou sucreries & $\begin{array}{l}\text { sucre, miel, confiture, gâteaux, bonbons, cookies, } \\
\text { biscuits et autres sucreries (boissons sucrées) }\end{array}$ & 0,5 \\
\hline 16 & Epices / condiments & $\begin{array}{c}\text { thé, café / chocolat, sel, ail, épices, levures, sauce } \\
\text { tomate, viandox, }\end{array}$ & $\mathbf{0}$ \\
\hline
\end{tabular}

Source: Ndiaye (2014)

Interprétation du SCAM: Pour interpréter se référer au tableau 6 :

Tableau 6. Seuils des scores de consommation alimentaire SCA

\begin{tabular}{|c|c|}
\hline Valeurs Seuils du SCAM & Indicateurs \\
\hline $\mathrm{SCAM} \leq 21$ & Consommation alimentaire pauvre \\
\hline $\mathrm{Si} \quad 21<\mathrm{SCAM} \leq 35$ & Consommation alimentaire limite \\
\hline $\mathrm{Si} \quad 35<\mathrm{SCAM} \leq 45$ & Consommation alimentaire moyennement acceptable \\
\hline SCA $>45$ & Consommation alimentaire acceptable \\
\hline
\end{tabular}

Source : Michikpe et al. (2017)

\section{Part des Dépenses Alimentaires (PDA) du ménage}

La Part des Dépenses Alimentaires (PDA) est un indicateur permettant d'estimer la vulnérabilité économique des ménages (Paridaens et al., 2017). Il 
est simplement construit en divisant le total des dépenses alimentaires par le total des dépenses du ménage. Cependant, le dénominateur et le numérateur doivent inclure la valeur des aliments consommés mais qui n’ont pas été achetés. Inclure les aliments non achetés dans le calcul de ce indicateur, donne la possibilité de faire entrer les ménages qui sont hautement dépendants des aliments non achetés dans la catégorie de ceux vulnérables économiquement. Si la valeur des aliments non achetés n'était pas prise en compte, de nombreux ménages pauvres qui sont très dépendent des aliments qu'ils produisent euxmêmes auraient moins de chance d'être classés comme vulnérables économiquement(VAM, 2014).

$$
=\frac{D A M}{D T M} \times 100 \quad \text { avec }\left\{\begin{array}{l}
P D A=\text { Part des Depenses Alimentaires } \\
D A M=\text { Dépenses Alimentaires du Ménage } \\
D M T=\text { Dépense total du Ménage }
\end{array}\right.
$$

Interprétation de PDA: La baisse de la part de l'alimentation dans les dépenses des ménages pourrait être vue comme une évolution positive parce que traduisant une amélioration des conditions économiques des ménages(DALODE et al., 2018). Elle peut ainsi être classée en quatre catégories de vulnérabilité économique (Tableau 7).

Tableau 7. Valeurs seuils de la Part des dépenses Alimentaires

\begin{tabular}{|c|c|}
\hline $\begin{array}{c}\text { Part de Dépense } \\
\text { Alimentaire (PDA) }\end{array}$ & Interprétations \\
\hline $\mathrm{Si} \mathrm{PDA}<50 \%$ & Vulnérabilité économique faible \\
\hline $\mathrm{Si} 50 \% \leq \mathrm{PDA}<65 \%$ & Vulnérabilité économique moyenne \\
\hline $\mathrm{Si} 65 \% \leq \mathrm{PDA}<75 \%$ & Vulnérabilité économique élevée \\
\hline $\mathrm{Si} \mathrm{PDA}>75 \%$ & Vulnérabilité économique très élevée \\
\hline
\end{tabular}

Source : Paridaens et Ndiayé (2017)

\section{Modélisation des précédents indicateurs de sécurité alimentaire}

Cette modélisation a permis de connaître l'influence des facteurs socio démographique et économiques sur les indicateurs de sécurité alimentaire. Le concept de sécurité alimentaire s'inscrit dans la philosophie économique issue du 'consensus de Washington', approche devenue dominante après le changement de paradigme intervenu dans la pensée économique (Azoulay, 2012). Le niveau d'accès alimentaire est fortement influencé par le revenu et le niveau socioéconomique(De Cock et al., 2013; Hackett et al., 2010; Isanaka et al., 2007; Maes et al., 2009; Regassa et al., 2012; Shariff et al., 2005; Tiyou et al., 2012). C'est pour mettre en exergue cette influence du revenu sur la sécurité alimentaire, que les $I n \_M N$; $I n \_P M L$ et $l n \_R B C$ des indicateurs de la rentabilité économique précédemment estimés seront aussi introduits comme variables explicatives dans la modélisation des indicateurs de sécurité 
alimentaire. Soit $\boldsymbol{I} \boldsymbol{S}_{\text {Alimi }} \mathbf{=} \mathbf{f}\left(\omega_{i}, \varepsilon_{i}\right)$ l'indicateur 'i' de sécurité alimentaire; on a :

$$
I S_{\text {Alim }} i=\mathbf{f}\left(\omega_{i}, \varepsilon_{i}\right)=\mu_{0}+\sum_{i=1}^{n} \mu_{i} . \omega_{i}+\varepsilon_{i}
$$

De façon détaillée on a :

$$
\begin{aligned}
& \boldsymbol{I S}_{\text {Alim }} \boldsymbol{i}=\mu_{0}+\mu_{1} \cdot \boldsymbol{S E X}+\mu_{2} \cdot \boldsymbol{A G E}+\mu_{3} \cdot \boldsymbol{N b r}_{\text {ACTIF }}+\mu_{4} \cdot \boldsymbol{N b r e}_{\text {An }_{\text {Scol }}}+ \\
& \mu_{5} \cdot \ln \_\boldsymbol{M N}+\mu_{6} \cdot \ln \_\boldsymbol{R B C}+\mu_{7} \cdot \ln \_\boldsymbol{P} \boldsymbol{M L}+\mu_{8} . \boldsymbol{A C C R E D I T}+\mu_{9} \cdot \mathbf{E m}+ \\
& \mu_{10} . \boldsymbol{H}_{\text {INSTR }}+\mu_{11} . \boldsymbol{C m}_{\text {DJOUG }}+\mu_{12} . \mathbf{C m}_{\text {KALAL }}+\mu_{13} . \boldsymbol{C m}_{\text {MALAN }}+\mu_{13} . \mathbf{D M R} \\
& + \\
& \mu_{14} . \boldsymbol{F A} \boldsymbol{A} \boldsymbol{B} \boldsymbol{A}+\mu_{15} . \boldsymbol{E V} \boldsymbol{D} \boldsymbol{T}+\mu_{16} . \boldsymbol{T Z P B}+\mu_{17} .2000 \boldsymbol{s y n}+\varepsilon_{i}
\end{aligned}
$$

Où :

* IS $S_{\text {Alim }} i$ est l’indicateur de sécurité alimentaire du producteur de maïs i ;

* $\omega_{i}$ les facteurs socio démographiques qui expliquent les différences de performance économique des producteurs de maïs ou les variables explicatives du modèle.

\begin{tabular}{|c|c|c|c|c|c|}
\hline Noms des variables & Codes & Modalités & $\begin{array}{c}\text { Signes } \\
\text { attendus } \\
\text { sur le } \\
\text { SDAM } \\
\end{array}$ & $\begin{array}{c}\text { Signes } \\
\text { attendus } \\
\text { sur le } \\
\text { SCAM } \\
\end{array}$ & $\begin{array}{l}\text { Signes } \\
\text { attendus } \\
\text { la PDA }\end{array}$ \\
\hline Genre de l'enquêté & SEX & $\begin{array}{c}1=\text { Masculin ; } \\
0=\text { Femme }\end{array}$ & - & - & + \\
\hline Age de l'enquêté & AGE & 1 & - & - & \pm \\
\hline Nombre d'actifs dans le ménage & Nbr_ACTIF & 1 & + & + & - \\
\hline Nbre Année de scolarisation & Nbre_An_Scol & 1 & + & + & - \\
\hline ln_Marge nette & ln_MN & 1 & \pm & \pm & - \\
\hline ln_Ratio Bénéfice coût & ln_RBC & 1 & + & + & - \\
\hline $\begin{array}{c}\text { ln_Productivité moyenne de main } \\
\text { d'œuvre }\end{array}$ & ln_PML & I & + & + & - \\
\hline Accès au crédit agricole & ACCREDIT & Non $=0 ;$ Oui $=1$ & + & + & \pm \\
\hline $\begin{array}{c}\text { Emigration pour de nouvelles } \\
\text { connaissances dans la culture du } \\
\text { maïs (JIMG) }\end{array}$ & Emg & Non $=0$; Oui $=1$ & \pm & \pm & - \\
\hline Instruits & H_INSTR & Non $=0 ;$ Oui $=1$ & + & + & - \\
\hline Commune de Djougou & Cm_DJOUG & Non $=0 ;$ Oui $=1$ & + & + & - \\
\hline Commune de Kalalé & Cm_KALAL & Non $=0$; Oui $=1$ & + & + & - \\
\hline Commune de Malanville & Cm_MALAN & Non $=0 ;$ Oui $=1$ & + & + & - \\
\hline
\end{tabular}

* $\mu_{i}$ les paramètres à estimer ou coefficients des variables explicatives.

* $\varepsilon_{i}$ sont les termes d'erreur aléatoires.

Les variables introduites dans le modèle sont détaillées dans le tableau suivant :

Tableau 8. Codes et modalités des variables explicatives du modèle de régression 


\begin{tabular}{|c|c|c|c|c|c|}
\hline Variété DMR.ESR.W & DMR & Non $=0$; Oui $=1$ & + & + & - \\
\hline Variété FAABA.obatampa. & FAABA & Non $=0$; Oui $=1$ & + & + & - \\
\hline Variété EV.DT.97.STR.W & EVDT & Non $=0$; Oui $=1$ & + & + & - \\
\hline Variété TZPB.SR.W & TZPB & Non $=0$; Oui $=1$ & + & + & - \\
\hline Variété 2000.syn.EE.W & 2000syn & Non $=0$; Oui $=1$ & + & + & - \\
\hline
\end{tabular}

Source : Auteur à partir des données d'enquête 2020

\section{Résultat}

\section{Estimation des indicateurs de la sécurité alimentaire}

Les ANOVA réalisées entre chaque indicateur et les différentes variétés améliorées de maïs nous permettent de déterminer si les moyennes de chacun de ces indicateurs (HHS, SDAM, SCAM et PDA) issues de l'adoption des variétés sont significativement différentes entre chacun des 5 groupes de variétés adoptées (2000 syn EE W, DMR, EVD, FAABA, TZPB) et les semences locales. Les données obtenues sont présentées sous formes de moyenne+/- écart type. (tableau 9)

\section{Indice domestique de faim ou Household Hunger Scale (HHS)}

Les résultats obtenus révèlent que l'indice catégorique de faim (HHS) de tous les producteurs est en moyenne nul. Les HHS des producteurs ne diffèrent pas.

\section{Score de Diversité Alimentaire des Ménage(SDAM)}

L'analyse de ce tableau révèle que la SDAM issue de l'adoption de chaque variété était statistiquement significativement différente entre chaque groupe de variété, $F=9,996$ et $[\operatorname{Pr}(>F)]<0.005$. La moyenne du SDAM de production des producteurs de la variété locale de maïs est de 3,62, inférieure à celle de chacune des variétés améliorées. Au niveau des variétés améliorées, la variété 2000Syn présente le meilleur et supérieur SDAM moyen (5,27 +/$1,11)$. Elle est suivie de la variété FAABA $(5,02+/-1,95)$. Cette moyenne a aussi baissé pour la variété DMR ESR W $(4,52)$ et celle du TZPB $(4,39)$. Enfin, la variété EVDT97STR vient en dernière position avec SDAM moyenne de 4,35+/- 0,9. En outre tous les adoptants des variétés améliorées ont un score de diversité alimentaire supérieur à 4 , par contre inférieure à 4 pour les non-adoptants (semences locales).

\section{Score de Consommation Alimentaire (SCAM)}

Le SCAM issue de l'adoption de chaque variété était significativement différente entre chaque groupe de variété, $\mathrm{F}=13,29$ et $[\operatorname{Pr}(>\mathrm{F})]<0.005$. La moyenne du SCAM des producteurs de la variété locale de maïs est de 25,65 unités, inférieure à celle de chacune des variétés améliorées. Au niveau des variétés améliorées, la variété DMR ESR W présente le meilleur et supérieur SCAM moyen (41,78 +/- 14,78). Elle est suivie de la variété 2000Syn (37,91 
+/- 14,3). Cette moyenne a aussi baissé pour la variété EVDT97STR $(37,65)$ et celle de TZPB $(29,53)$. Enfin, la variété FAABA vient en dernière position avec un SCAM moyen de 28,67+/- 10,21.

\section{Part des Dépenses Alimentaire (PDA)}

La PDA issue de l'adoption de chaque variété était statistiquement significativement différente entre chaque groupe de variété, $F=13,29$ et $[\operatorname{Pr}(>\mathrm{F})]<0.005$. La moyenne de la PDA des producteurs de la variété locale de maïs est de 63,65 unités, supérieure à celle de chacune des variétés améliorées. Au niveau des variétés améliorées, la variété FAABA présente la PDA moyenne la plus élevée (61,33 +/-16,87). La variété 2000 Syn vient en deuxième position $(59,31+/-18,58)$. Elle est suivie de la variété EVDT97STR $(57,37+/-18,16)$. Cette moyenne a aussi baissé pour la variété DMR ESR W $(50,82)$ et celle du TZB $(47,66)$. Autrement dit les ménage adoptant les VAM ont en moyenne des PDA inférieures à celles des non adoptants.

Tableau 9. ANOVA entre les indicateurs de sécurité alimentaire et les variétés de maïs

\begin{tabular}{|c|c|c|c|c|}
\hline Variétés & HHS & SDAM & SCAM & PDA \\
\hline \multirow{2}{*}{ 2000synEEW } & 0 & $\mathbf{5 , 2 6}$ & $\mathbf{3 7 , 9 1}$ & $\mathbf{5 9 , 3}$ \\
\cline { 2 - 5 } & & $(1,11)$ & $(14,82)$ & $(18,58)$ \\
\hline \multirow{2}{*}{ DMR ESR W } & 0 & $\mathbf{4 , 5 1}$ & $\mathbf{4 1 , 7 7}$ & $\mathbf{5 0 , 8 1}$ \\
\cline { 2 - 5 } & & $(0,80)$ & $(14,77)$ & $(18,27)$ \\
\hline \multirow{2}{*}{ EVDT97STR } & 0 & $\mathbf{4 , 3 5}$ & $\mathbf{3 7 , 6 4}$ & $\mathbf{5 7 , 3 6}$ \\
\cline { 2 - 5 } & & $(0,87)$ & $(13,19)$ & $(18,16)$ \\
\hline \multirow{2}{*}{ FAABA.Obatampa } & 0 & $\mathbf{5 , 0 2}$ & $\mathbf{2 8 , 6 6}$ & $\mathbf{6 1 , 3 3}$ \\
\cline { 2 - 5 } & & $(1,95)$ & $(10,21)$ & $(16,87)$ \\
\hline \multirow{2}{*}{ TZPB SRW } & 0 & $\mathbf{4 , 3 8}$ & $\mathbf{2 9 , 5 3}$ & $\mathbf{4 7 , 6 5}$ \\
\cline { 2 - 5 } & & $(1,57)$ & $(12,01)$ & $(13,96)$ \\
\hline \multirow{2}{*}{ Locale } & 0 & $\mathbf{3 , 6 2}$ & $\mathbf{2 5 , 6 5}$ & $\mathbf{6 3 , 6 5}$ \\
\hline ANOVA & - & $\mathrm{F}=9,996$ & $\mathrm{~F}=13,29$ & $\mathrm{~F}=7,837$ \\
& & $\mathrm{p}=0,0000$ & $\mathrm{p}=9,36 \mathrm{e}-12$ & $\mathrm{p}=0,0000$ \\
\hline
\end{tabular}

Source : Auteur à partir des données d'enquête 2020

\section{Déterminants des indicateurs de sécurité alimentaire Description des variables introduites dans les modèles}

Le tableau 10 présente la description des différentes variables explicatives introduites dans les modèles. Les enquêtés sont majoritairement des hommes (65,31\%) âgés de 41 ans en moyenne. Ces enquêtés sont les producteurs de maïs issus des Communes de Malanville (69), de Banikoara (81), de Kalalé (94) et de Djougou (76). Le nombre moyen d'années de scolarisation de ces enquêtés est de 7 ans avec un taux d'instruction de $28 \%$. En dépit de ce faible taux d'instruction, seulement $22 \%$ de ces producteurs ont une fois au moins émigré afin d'acquérir de nouvelles connaissances sur les techniques culturales de la production de maïs. Le nombre d'actifs dans 
leur ménage est environ deux (2) personnes avec une forte dispersion au tour de la moyenne. Malgré que c'est seulement $18 \%$ des enquêtés qui ont accès au crédit, $78 \%$ ont adopté les variétés améliorées de maïs. Ainsi, 12,81 \% ; $28,12 \% ; 8,44 \% ; 9,69 \%$ et 18,44 \% sont respectivement les taux d'adoption de variétés améliorées 2000synEEW; FAABA.Obatampa; DMRESRW; EVDT97STRW et TZPBSRW. L'estimation de la rentabilité économique de la production du maïs révèle que les moyennes de la Marge Nette (MN), de la Productivité Moyenne de main d'œuvre (PML) et du Ratio Bénéfice Coût (RBC) des producteurs des variétés améliorées de maïs sont respectivement 239 935,90; 2 660,51 et 1,44. Tandis qu'elles sont respectivement de 63 842,$46 ; 1434,36$ et 0,96 pour les producteurs des variétés locales.

Tableau 10. Statistiques descriptives des variables introduites dans le modèle

\begin{tabular}{|c|c|c|c|}
\hline \multicolumn{2}{|c|}{ Variables Quantitatives } & Moyenne & Ecart-type \\
\hline \multicolumn{2}{|l|}{ Age de l'enquêté } & 41,34 & 12,07 \\
\hline \multicolumn{2}{|c|}{ Nbre d’actifs dans le ménage } & 1,86 & 4,07 \\
\hline \multicolumn{2}{|c|}{ Nbre Année de scolarisation } & 7,04 & 2,13 \\
\hline \multirow[t]{3}{*}{ Marge nette } & Globale & 200314,87 & 274600,86 \\
\hline & Adoptants & 239935,90 & 263581,6 \\
\hline & Non Adoptants & 63842,46 & 26968,18 \\
\hline \multirow[t]{3}{*}{ Ratio Bénéfice coût } & Global & 1,33 & 1,02 \\
\hline & Adoptants & 1,44 & 1,04 \\
\hline & Non Adoptants & 0,96 & 0,85 \\
\hline \multirow{3}{*}{$\begin{array}{c}\text { Productivité moyenne de main } \\
\text { d'œuvre }\end{array}$} & Globale & 2384,62 & 2124,29 \\
\hline & Adoptants & 2660,51 & 2042,79 \\
\hline & Non Adoptants & 1434,36 & 2139,18 \\
\hline \multirow[t]{3}{*}{ Variables Qualitatives } & Modalités & Effectifs & $\begin{array}{c}\text { Pourcentage } \\
(\%)\end{array}$ \\
\hline & Oui & 72 & 22,5 \\
\hline & Non & 248 & 77,5 \\
\hline \multirow[t]{2}{*}{ Adoption de la variété 2000synEEW } & Non & 279 & 87,19 \\
\hline & Oui & 41 & 12,81 \\
\hline \multirow{2}{*}{$\begin{array}{l}\text { Adoption de la variété } \\
\text { FAABA.Obatampa }\end{array}$} & Non & 230 & 71,88 \\
\hline & Oui & 90 & 28,12 \\
\hline \multirow[t]{2}{*}{ Adoption de la variété DMRESRW } & Non & 293 & 91,56 \\
\hline & Oui & 27 & 8,44 \\
\hline \multirow{2}{*}{$\begin{array}{l}\text { Adoption de la variété } \\
\text { EVDT97STRW }\end{array}$} & Non & 289 & 90,31 \\
\hline & Oui & 31 & 9,69 \\
\hline \multirow[t]{2}{*}{ Adoption de la variété TZPBSRW } & Non & 261 & 81,56 \\
\hline & Oui & 59 & 18,44 \\
\hline \multirow[t]{2}{*}{ Genre de l'enquêté } & Masculin & 209 & 65,31 \\
\hline & Féminin & 111 & 34,69 \\
\hline \multirow[t]{4}{*}{ Commune } & Banikoara & 81 & 25,31 \\
\hline & Djougou & 76 & 23,75 \\
\hline & Kalalé & 94 & 29,38 \\
\hline & Malanville & 69 & 21,56 \\
\hline Accès au crédit & Non & 260 & 81,25 \\
\hline
\end{tabular}




\begin{tabular}{|c|c|c|c|}
\hline & Oui & 60 & 18,75 \\
\hline \multirow{2}{*}{$\begin{array}{c}\text { Emigration pour l'agriculture } \\
\text { (JIMG) }\end{array}$} & Non & 248 & 77,5 \\
\cline { 2 - 4 } & Oui & 72 & 22,5 \\
\hline Instruits & Oui & 88 & 28 \\
\cline { 2 - 4 } & non & 232 & 73 \\
\hline
\end{tabular}

Source: Auteur à partir des données d'enquête 2020

Modélisation des indicateurs de sécurité alimentaire (Tableau 11)

Les trois (03) modèles sont globalement significatifs au seuil de $1 \%$ (p-value $=0.000$ ). Respectivement $23,24 \%, 24,41 \%$ et $26,05 \%$ de la variation du Score de Diversité Alimentaire (SDAM); de Consommation Alimentaire (SCAM) et de la Part des Dépenses Alimentaire (PDA) étaient expliquées par les variations des variables exogènes introduites dans les modèles.

Facteurs sociodémographiques : Le fait que le producteur soit un homme influence négativement le SCAM avec une baisse allant juste à 3,07 unités. Alors qu'il baisse de 0,12 lorsque l'âge du producteur augmente d'un an. Cependant l'appartenance à la Commune de Djougou, comparativement à celle Banikoara augmente le SCAM de 3,79.

L'augmentation supplémentaire d'un membre actif dans le ménage augmente la part des dépenses alimentaires (PDA) de 0,67 tandis qu'elle se réduit de 6,91 au fur et à mesure que le nombre d'année de scolarisation du producteur augmente. En outre l'appartenance à la Commune de Kalalé, comparativement à celle de Banikoara augmente la PDA de 13,30.

Facteurs socioéconomiques : l'émigration pour acquérir de nouvelles connaissances dans la production du maïs réduit le SDAM et la PDA respectivement de 0,77 et de 8 .

L'augmentation d'une unité de la productivité moyenne de la main d'œuvre familiale augmente le SCAM de 4,60\%. Alors que la réduction d'un franc de la marge nette due à l'alimentation de cette main d'œuvre familiale entraîne une augmentation de 0,71\% du SDAM. Dans le même temps une augmentation supplémentaire du Ratio Bénéfice Coût réduit la PDA de 9,23\%. Comparativement aux variétés locales l'adoption des variétés améliorées de maïs influence positivement le SDAM et le SCAM et négativement la PDA. Ainsi les augmentations respectives du SDAM et du SCAM est de 1,21 et 15,58 lorsque le producteur adopte la DMR ; elles sont de 1,71 et de 5,69 pour la FAABA ; de 0,94 et de 13,73 pour l'adoption de la EVDT ; elle est par contre de 1,19 et de 7,92 quand le producteur adopte la TZPB et enfin de 1,94 et de 12,99 lorsque le producteur à opter pour la 2000syn. Cependant l'adoption de DMR EVDT et TZPB diminue respectivement la PDA de 9,11; de 9,73 et de 16,00 . 
Tableau 11. Modélisation des indicateurs de la sécurité alimentaire

\begin{tabular}{|c|c|c|c|c|c|c|}
\hline \multirow[t]{2}{*}{ Variables } & \multicolumn{2}{|c|}{ SDAM } & \multicolumn{2}{|c|}{ SCAM } & \multicolumn{2}{|c|}{ PDA } \\
\hline & Coeff & $\begin{array}{l}\text { Erreurs } \\
\text { types }\end{array}$ & Coeff & $\begin{array}{c}\text { Erreurs } \\
\text { types }\end{array}$ & Coeff & $\begin{array}{c}\text { Erreurs } \\
\text { types }\end{array}$ \\
\hline Constante & $7.460 * * *$ & 1.542 & $28.143 * *$ & 12.799 & $56.980 * * *$ & 17.181 \\
\hline SEX & -0.087 & 0.185 & $-3.072 * *$ & 1.535 & 1.009 & 2.061 \\
\hline ln_MN & $-0.710 * *$ & 0.303 & -3.203 & 2.515 & -0.779 & 3.376 \\
\hline In_RBC & 0.137 & 0.404 & -0.711 & 3.357 & $-9.234 * *$ & 4.507 \\
\hline In_PML & -0.045 & 0.340 & 4.609** & 2.825 & 2.929 & 3.793 \\
\hline H_INSTR & -0.018 & 0.323 & 4.366 & 2.680 & -3.632 & 3.598 \\
\hline Nbre_An_Scol & 0.296 & 0.202 & 1.661 & 1.681 & $-6.915 * * *$ & 2.257 \\
\hline Cm_DJOUG & 0.346 & 0.273 & 3.797* & 2.270 & 4.814 & 3.047 \\
\hline Cm_KALAL & 0.157 & 0.277 & -0.617 & 2.302 & $13.304 * * *$ & 3.090 \\
\hline Cm_MALAN & 0.133 & 0.290 & 0.9762 & 2.409 & 3.678 & 3.234 \\
\hline AGE & -0.008 & 0.007 & $-0.121 *$ & 0.061 & 0.003 & 0.083 \\
\hline Nbr_ACTIF & -0.0204 & 0.036 & -0.279 & 0.299 & 0.679* & 0.402 \\
\hline Emg & $-0.773 * * *$ & 0.229 & -2.149 & 1.902 & $-8.001 * * *$ & 2.554 \\
\hline ACC & 0.345441 & 0.277 & -1.893 & 2.299 & 0.528 & 3.086 \\
\hline DMR & $1.213^{* * *}$ & 0.380 & $15.580 * * *$ & 3.155 & $-9.114 * *$ & 4.235 \\
\hline FAABA & $1.710 * * *$ & 0.277 & $5.698 * *$ & 2.305 & -2.172 & 3.094 \\
\hline EVDT & $0.946 * *$ & 0.370 & $13.733 * * *$ & 3.073 & $-9.739 * *$ & 4.126 \\
\hline TZPB & $1.190 * * *$ & 0.314 & $7.920 * * *$ & 2.609 & $-16.006 * * *$ & 3.502 \\
\hline 2000syn & $1.941 * * *$ & 0.333 & $12.991 * * *$ & 2.766 & -0.253 & 3.713 \\
\hline $\begin{array}{c}\text { Nombre } \\
\text { d'observation }\end{array}$ & \multicolumn{2}{|c|}{$\begin{array}{c}\text { Multiple R-squared: } \\
0,2324\end{array}$} & \multicolumn{2}{|c|}{$\begin{array}{c}\text { Multiple R-squared: } \\
0,2441\end{array}$} & \multicolumn{2}{|c|}{$\begin{array}{c}\text { Multiple R-squared: } \\
0,2605 \\
\end{array}$} \\
\hline 320 & \multicolumn{2}{|c|}{$\begin{array}{c}\text { Adjusted R-squared: } \\
0,1786 \\
\end{array}$} & \multicolumn{2}{|c|}{$\begin{array}{c}\text { Adjusted R-squared: } \\
0,1912 \\
\end{array}$} & \multicolumn{2}{|c|}{$\begin{array}{c}\text { Adjusted R-squared: } \\
0,2087 \\
\end{array}$} \\
\hline & \multicolumn{2}{|c|}{$\begin{array}{l}\text { F-statistic: 4,323 on } \\
18 \text { and } 257 \mathrm{DF},\end{array}$} & \multicolumn{2}{|c|}{$\begin{array}{l}\text { F-statistic: 4,611 on } \\
18 \text { and } 257 \mathrm{DF},\end{array}$} & \multicolumn{2}{|c|}{$\begin{array}{c}\text { F-statistic: } 5,03 \text { on } 18 \\
\text { and } 257 \mathrm{DF} \text {, }\end{array}$} \\
\hline & \multicolumn{2}{|c|}{$\begin{array}{c}\text { p-value: } \\
0,00000004467\end{array}$} & \multicolumn{2}{|c|}{$\begin{array}{c}\text { p-value: } \\
0,000000009063\end{array}$} & \multicolumn{2}{|c|}{ p-value: 8,893e-10 } \\
\hline
\end{tabular}

Source: Auteur à partir des données d'enquête 2020

\section{Discussion}

A la lumière des résultats, le HHS de tous les producteurs est compris entre 0 et 1 ce qui signifie qu'aucun ménage producteur de maïs n'est resté plusieurs jours affamé. Ce résultat va dans le même sens que celui des travaux du PNUD (2015) sur la sécurité alimentaire et le développement humain qui stipule qu'en vingt-cinq ans, la prévalence de la faim a été réduite de moitié au Bénin, passant de 22,5\% en 1990 à 11,2\% en 2014. Selon le même rapport du PNUD le Bénin est parvenu à se hisser à la deuxième place des pays où la faim est moins prononcée en Afrique de l'Ouest. L'adoption ou non des variétés améliorées de maïs n’a pas d’influence sur la faim au Bénin. En effet, la réduction de la faim au Bénin est fondamentalement due aux mesures prises en faveur de la mère et de l'enfant dans le cadre de la lutte contre le paludisme, l'accroissement de la part des naissances dans les centres médicaux et la 
meilleure prise en charge prénatale (PNUD, 2015). Mais idéalement, l'indice domestique de faim (HHS) ne doit pas être utilisé comme une mesure unique et autonome de l'insécurité alimentaire, mais plutôt comme une partie d'une série d'outils pour mesurer les aspects complémentaires de l'insécurité alimentaire (Ballard, 2011). Ainsi l'évaluation de la diversité et de la consommation alimentaire a révélé que le score de diversité alimentaire de tous les adoptants des variétés améliorées de maïs est supérieure à 4 (la norme fixée par le PNUD) par contre la moyenne du SDAM des non adoptants est de 3,62 inférieure à 4. Les adoptants des variétés améliorées de maïs ont alors une alimentation plus diversifiée que celle des non adoptants. Les non adoptants des VAM et les adoptants des variétés améliorées de maïs telles que la FAABA.Obatampa et la TZPB SRW ont en moyenne des SCAM compris entre 21 et 35. Alors ils ont une consommation alimentaire limite. Dans le même temps les adoptants de la 2000synEEW, DMR ESR W et de la EVDT97STR ont une consommation alimentaire moyennement acceptable pour avoir totalisé des SCAM compris entre 35 et 45 . Toutefois il est à noter que le plus faible SCAM a été enregistré dans le rang des non adoptants des VAM. Constituée essentiellement des aliments de base (céréales/tubercules) avec un peu de légumes et d'huile, l'alimentation des ménages non adoptants comparativement à ceux des adoptants des VAM est pauvre et très peu diversifiée. Malgré que la part du budget consacré à l'alimentation par les ménages en milieu rural a diminué, passant de 51 à 47\% en 2017 (DALODE et al., 2018) au Bénin, exceptés les ménages producteurs de la variété améliorée TZPB SR W qui ont une vulnérabilité économique faible car leur Part des Dépenses Alimentaires est égale à $47,65 \%<$ donc à $50 \%$, tous les autres ménages producteurs de maïs ont des PDA $>50 \%$. Ces ménages dépensent donc plus de $50 \%$ de leur budget dans l'alimentation. Malgré ces résultats peu satisfaisant, il est à noter que la PDA la plus élevée (63,65\%) a été enregistrée au sein des ménages non adoptants, ils sont donc économiquement plus vulnérables que les ménages ayant adopté les VAM. Le revenu issus de la production de maïs garanti significativement la sécurité alimentaire du ménage. Ces différents indicateurs de la sécuritaire alimentaire sont influencés par des facteurs socio-économiques et démographiques.

Ainsi, en passant d'un producteur de sexe féminin à un producteur de sexe masculin le score de consommation et de diversité alimentaire diminue. Ce qui est normal car les hommes en tant que père de famille ont plus de personnes à nourrir à charge que des femmes qui, pour la plupart, sont nourries par leurs maris. Ces résultats convergent avec plusieurs autres études concernant les liens entre genre et alimentation au sein des ménages. Hoddinott et al. (1995) puis Duflo et al. (2004) en Côte d'Ivoire, et Doss (2005) au Ghana ont montré que le pouvoir de décision des femmes et leur contrôle sur les ressources sont reliés à l'allocation pour l'alimentation d'une 
plus grande part des revenus ; Malapit et al. (2015) et Lourme-Ruiz et al. (2016) au Népal ont établi que les femmes ont un meilleur score de diversité alimentaire et des enfants en meilleur état nutritionnel quand elles ont plus de revenus et d'autonomie dans la décision, et/ou quand elles vivent dans des ménages où les pouvoirs entre hommes et femmes sont plus équilibrés. C'est pourquoi, les femmes gérantes de la production du maïs ont plus de chance d'avoir une sécurité alimentaire plus améliorée que celle des producteurs masculins.

Les zones agro-écologiques expliquent d'une certaine manière la sécurité alimentaire des ménages producteurs de maïs. En analysant le score consommation alimentaire, les résultats permettent de conclure que les ménages des producteurs de la Commune de Djougou comparativement à ceux de Banikoara ont des scores de consommation alimentaire plus élevés. Paradoxalement, c'est la Commune de Kalalé qui est plus vulnérable économiquement que celle de Banikoara. Ces résultats abondent dans le même sens que celui de Guindo et al. (2018) sur la comparaison du profil de sécurité alimentaire des ménages dans les régions de Gao et Sikasso au Mali qui a montré que la situation de la sécurité alimentaire varie d'une région à une autre. Cette situation révèle la difficulté qu'éprouvent certains producteurs à livrer leur output à cause des voies d'accès dans la Commune.

Plus les producteurs sont scolarisés plus la sécurité alimentaire de leurs ménages est améliorées. Ceci est dû au fait qu'ils sont plus organisés que les non scolarisés. En outre ils auraient appris à l'école que la consommation et les variations des mets, permettant de procurer des aliments simples, minéraux et des vitamines requis à l'organisme lui sont bénéfiques. Ces résultats corroborent ceux de Ahmadi et al. (2019). Ces auteurs ont démontré qu'un faible niveau d'instruction engendre l'insécurité alimentaire. Alors qu'un an plus tôt, Ajayi et al. (2018) avaient prédit que le niveau d'éducation améliore le niveau d'efficacité technique des producteurs qui sera un antidote au problème de l'insécurité alimentaire.

Plus l'âge du producteur augmente plus les personnes à charge et le nombre d'actif dans le ménage augmentent. Or un ménage qui a plus d'actifs voit son score de consommation alimentaire diminué (Akinloye et al., 2016). C’est pourquoi dans la majorité des études, l'âge a été associé à un accès alimentaire plus faible (Chatterjee et al., 2012; Hackett et al., 2010; Isanaka et al., 2007; Leyna et al., 2010; Nagata et al., 2012; Tsai et al., 2011). En outre, cette vulnérabilité a été attribuée à une diminution de l'état de santé des individus et à une diminution de leur capacité d'effectuer des travaux physiques associés à l'agriculture (Nagata et al., 2012). C'est pourquoi, l'âge et le nombre d'actif exercent une influence négative sur la sécurité alimentaire du ménage. 
Les mains d'œuvre familiales générées par ces membres actifs du ménage engendrent des coûts supplémentaires liés à l'alimentation des membres du ménage. En effet, l'expérience a prouvé que plus on exerce des activités physiques au champ plus on consomme d'aliment. Alors un coût supplémentaire lié à l'alimentation du ménage se crée. Pour une bonne estimation de la marge nette, ce coût lié à l'alimentation a été amputé de la recette. Cette amputation réduit la marge nette mais améliore le score de consommation ou de diversité alimentaire. C'est la raison pour laquelle la réduction d'un franc de la marge nette due à l'alimentation de cette main d'œuvre familiale entraîne une augmentation de 0,0071 unité du SDAM. Et c'est pourquoi, dans le même temps l'augmentation d'un 1 franc de la productivité moyenne de main d'œuvre familiale augmente le score de consommation alimentaire de 4,60\%. Lorsque le Ratio Bénéfice Coût augmente d'un franc la vulnérabilité économique du ménage se réduit de 9,23\%. Ces résultats nous laissent dire que l'amélioration de la sécurité alimentaire du ménage épuise le revenu de la production tout en augmentant les dépenses alimentaires du ménage. Pour Bocoum (2012), l’accroissement des dépenses totales (considérées comme proxy des revenus) améliore quantitativement (calories) et qualitativement (diversité) la consommation alimentaire des ménages, confirmant ainsi les résultats de cette recherche.

Etant donné que les adoptants des VAM ont une différence significative positive de la moyenne, comparativement aux non adoptants, il est donc normal que l'adoption des cinq variétés améliorées spécialement par les producteurs rend résilient leur ménage face à l'insécurité alimentaire à travers le SDAM, SCAM et la PDA. Ce résultat vient confirmer celui des travaux de Temple et al. (2015) qui stipule que le renforcement des capacités d'innovations locales permet de réaliser les potentialités des agrosystèmes pour la sécurité alimentaire. Et celui Lourme-Ruiz et al. (2016) pour qui, augmenter la production agricole peut théoriquement améliorer la diversité de l'alimentation, via l'autoconsommation.

Contre toute attente, le fait d'émigrer pour acquérir de nouvelles connaissances dans la production du maïs exerce une influence négative sur le score de diversité alimentaire. Si jusque-là cette étude nous a prouvé que l'adoption des variétés améliorées réduit l'insécurité alimentaire du ménage, alors les formations reçues par les producteurs émigrés auraient incité la majorité à produire les variétés locales. Du coup les formations qu'ils avaient reçues ne sont pas en adéquation avec les réalités de leur localité. Par ailleurs, la migration pour des fins agricole réduit l'effectif de la main d'œuvre familiale et peut diminuer par conséquent la productivité agricole du ménage (Lebrun, 2013). Or pour Prskawetz et al. (2003) l'insécurité alimentaire, la faible productivité agricole et les inégalités dans la distribution des aliments sont positivement corrélées. 


\section{Conclusion}

Cette étude a montré, d'une part que l'insécurité alimentaire n’est pas sévère dans notre zone de recherche (au Nord-Bénin) et d'autre part que les ménage des producteurs ayant adopté au moins une des cinq variétés améliorées de maïs à savoir DMRESRW, FAABA.Obatampa, EVDT97 STR W, TZPB SR W et 2000Syn.EEW sont moins en insécurité alimentaire que les ménages des non adoptants (Producteurs des variétés locales de maïs). Donc l'adoption de ces variétés améliorées de maïs réduit l’insécurité alimentaire des ménages adoptants à travers l'augmentation des rendements et de la rentabilité économique de la production. Pour que la culture du maïs assure la sécurité alimentaire mieux que toute autre culture comme l'ont prédit les études antérieures de Baco et al. (2011), les gouvernants, projets et programmes doivent promouvoir davantage les variétés améliorées de maïs en générale et les cinq variétés améliorées adoptées dans cette zone de recherche en particulier. Et sensibiliser les producteurs sur les techniques culturales de ces variétés améliorées de maïs.

\section{References:}

1. Adekambi, A.S., Agbotridja, V.D., Hinnou, C.L., Kossoko, O.C.D., 2020. Impact de l'adoption des technologies resilientes sur le bien-etre des menages producteurs de maïs au Nord du Benin: Journal de la Recherche Scientifique de l’Université de Lomé 22(3), 21-39.

2. Affholder, F., Poeydebat, C., Corbeels, M., Scopel, E., Tittonell, P., 2013. The yield gap of major food crops in family agriculture in the tropics : Assessment and analysis through field surveys and modelling. Field Crops Research 143(March), 106-118.

3. Ahmadi, D., Melgar-Quiñonez, H., 2019. Determinants of food insecurity in occupied Palestinian territory: a cross-sectional survey. Lancet 393, S4.

4. Ajayi, C.O., Oluntumise, A.I., 2018. Determinants of food security and technical efficiency of cassava farmers in Ondo State, Nigeria. International Food and Agribusiness Management Review 21(10302019-602), 915-928.

5. Akinloye, O., Putuma, M., Adeyemi ADEYEFA, S., 2016. Determinants of Food Insecurity among the Urban Poor in the City of Tshwane, South Africa. Journal of Economics and Development Studies 4, 101-114. https://doi.org/10.15640/jeds.v4n2a9.

6. Akpo, C.Y., 2020. Impact des semences améliorées sur la productivité du maïs au Bénin. African Economic Research Consortium (AERC) E1a, 1-26.

https://aercafricahub.org/wp-content/uploads/2020/11/E1aChristelleYeba-Akpo-FRFrench.pdf (Consulté le 06/07/2021). 
7. Azoulay, G., 2012. Sécurité alimentaire mondiale et crise structurelle d'un mode de fonctionnement de l'économie agricole. L'Homme et la Société $n^{\circ}$ 183-184(1), 61-81.

8. Baco, M.N., Abdoulaye, T., Sanogo, D., Langyintuo, A., 2011. Caractérisation des ménages producteurs de maïs en zone de savane sèche au Bénin. Publication de l'INRAB -Bénin- IITA produite dans le cadre du Projet Maïs tolérant à la sécheresse (DTMA) pour l'Afrique Rapport pays-Enquête-ménage. 38p.

https://core.ac.uk/download/pdf/132689959.pdf; consulté le $16 / 01 / 2020$.

9. Ballard, T., 2011. Indice domestique de la faim: Définition de l'indicateur et guide de mesure 26.

10. Ballard, T., Coates, J., Swindale, A., Deitchler, M., 2011. Indice domestique de la faim: Définition de l'indicateur et guide de mesure. Washington, DC: Food and Nutrition Technical Assistance III Project, FHI 360.

11. Bocoum, I., 2012. Insécurité alimentaire au Mali. Identifier les ménages vulnérables avec précision. Perspective, CIRAD, $2012 \mathrm{~N}^{\circ}$ 14., 4 p. DOI: $10.18167 /$ agritrop/00020. https://doi.org/10.18167/agritrop/00020

12. Bucekuderhwa, C., Mapatano, S., 2013. Comprendre la dynamique de la vulnérabilité à l'insécurité alimentaire au Sud-Kivu. La Revue Électronique En Sciences de l'environnement 17, 31.

13. Chatterjee, N., Fernandes, G., Hernandez, M., 2012. Food insecurity in urban poor households in Mumbai, India. Food Security 4(4), 619632.

14. CORAF /WECARD, 2018. Impact de l'adoption des variétés améliorées de maïs sur le bien-être des maïsiculteurs au Bénin, au Burkina-faso, en Cote d'ivoire et au Mali. CENTRE NATIONAL DE SPECIALISATION SUR LE MAÏS (CNS-Maïs) 46p.

15. Dalode, E.A., Nago, M.C., Aoudji, A., Assogbadjo, A., Tossa, J.C., Paulin Azokpota, P., Koukoubou, E.N., 2018. Examen stratégique national <<Faim Zéro >> au Bénin à l'horizon 2030. Gouvernement Béninois et Programme Alimentaire Mondial (PAM-Bénin). 192p. https://docs.wfp.org/api/documents/WFP-0000114271/download/ (Consulté le 10/11/2020).

16. De Cock, N., D’Haese, M., Vink, N., van Rooyen, C.J., Staelens, L., Schönfeldt, H.C., D’Haese, L., 2013. Food security in rural areas of Limpopo province, South Africa. Food Security 5(2), 269-282.

17. Diouf Sarr, N.S., Basse, B.W., Fall, A.A., 2018. Taux et déterminants de l'adoption de variétés améliorées de riz au Sénégal. Économie rurale. Agricultures, alimentations, territoires 51-68. 
https://doi.org/10.4000/economierurale.5897

18. Doss, C., 2005. The effects of intrahousehold property ownership on expenditure patterns in Ghana. J Afr Econ 155(1), 149-180. DOI: 10.1093/jae/eji025.

19. Duflo, E., Udry, C., 2004. Intrahousehold resource allocation in Cote d'Ivoire: social norms, separate accounts and consumption choices. In: National Bureau of Economic Research Working PaperNo. 10498.

20. Dury, S., Bocoum, I., 2012. Le «paradoxe» de Sikasso (Mali): pourquoi «produire plus » ne suffit-il pas pour bien nourrir les enfants des familles d'agriculteurs? Cahiers Agricultures 21(5), 324-336.

21. Dury, S., Vall, É., Imbernon, J., 2017. Production agricole et sécurité alimentaire en Afrique de l'Ouest. Cah. Agric. 26, 61001. https://doi.org/10.1051/cagri/2017047

22. EPA, E.P.A., 2019. Utiliser les résultats de recherche pour améliorer la sécurité alimentaire et nutritionnelle en milieu urbain. Netherlands Organisation for Scientific Research 12p.

https://knowledge4food.net/wpcontent/uploads/2019/09/EPAforum_c oncept-note_fr.pdf consulté le 16/06/2021.

23. Gérard, F., Dury, S., Bélières, J.-F., Keita, M.S., Bénoit-Cattin, M., 2012. Comparaison de plusieurs scénarios de lutte contre l'insécurité alimentaire au Mali. Cahier Agriculture 21(5), 356-365.

24. Grethe, H., Luckmann, J., Siddig, K., Kinkpe, T., 2020. Analyse exante du 'Plan National d'Investissements Agricoles et de Sécurité Alimentaire et Nutritionnelle" du Benin. Deutsche Gesellschaft für Internationale Zusammenarbeit (GIZ) GmbH, Bonn et Eschborn, Allemagne; 72p.

https://www.snrd-africa.net/wp-content/uploads/2021/02/2021-0205_GIZ_PNIASAN_FR_web.pdf (Conslté le 06/07/2021).

25. Guindo, E., Diarra, S., Coulibaly, D., Diawara, F., Nina, N.K., Konate, F., Iknane, A.A., 2018. Comparaison du profil de sécurité alimentaire des ménages dans les régions de Gao et Sikasso en période de postrécolte au Mali. Mali Sante Publique 8(1), 18-21.

26. Hackett, M., Melgar-Quiñonez, H., Taylor, C.A., Uribe, M.C.A., 2010. Factors associated with household food security of participants of the MANA food supplement program in Colombia. Archivos Latinoamericanos De Nutricion 60(1), 42-47.

27. Headey, D., Ecker, O., 2013. Rethinking the measurement of food security: From first principles to best practice. Food Security 5(3), 327-343.

28. Hoddinott, J., Haddad, L., 1995. Does female income share influence household expenditures? Evidence from Côte d'Ivoire. Oxford: Oxford Bulletin of Economics and Statistics PP.77-95. 
29. INSAE, I.N. de la S. et de l'Analyse E., 2016. RGPH4: Cahier des villages et quartiers de ville du département de l'Alibori; du Borgou et du Donga. REPUBLIQUE DU BENIN: MINISTERE DU PLAN ET DU DEVELOPPEMENT.

https://insae.bj/images/docs/insae-statistiques/enquetesrecensements/RGPH/1.RGPH_4/resultats\%20finaux/Cahiers\%20villa ges/Cahier\%20des\%20villages\%20et\%20quartiers\%20de\%20ville\%2 0Alibori.pdf (Consulté le 17/06/2020).

30. Isanaka, S., Mora-Plazas, M., Lopez-Arana, S., Baylin, A., Villamor, E., 2007. Food insecurity is highly prevalent and predicts underweight but not overweight in adults and school children from Bogotá, Colombia. Journal of Nutrition 137(12), 2747-2755.

31. Jayne, T.S., Mather, D., Mghenyi, E., 2010. Principal Challenges Confronting Smallholder Agriculture in Sub-Saharan Africa. World Development 38(10), 1384-1398.

32. Jones, A.D., Shrinivas, A., Bezner-Kerr, R., 2014. Farm production diversity is associated with greater household dietary diversity in Malawi: Findings from nationally representative data. Food Policy 46, $1-12$.

33. Kayodé, A.P.P., 2018. Adoption de variétés de sorgho résilientes aux changements climatiques au Bénin. Le Centre technique de coopération agricole et rurale (CTA), Wageningen, les Pays-Bas. 36p. https://hdl.handle.net/10568/98382 (Consulté le 12/11/2020).

34. Lebrun, N., 2013. Commerce et discontinuités. Cahiers de géographie du Québec 58(163), 7-149.

35. Legwegoh, A.F., Hovorka, A.J., 2013. Assessing food insecurity in Botswana: The case of Gaborone. Development in Practice 23(3), 346-358.

36. Leyna, G.H., Mmbaga, E.J., Mnyika, K.S., Hussain, A., Klepp, K.I., 2010. Food insecurity is associated with food consumption patterns and anthropometric measures but not serum micronutrient levels in adults in rural Tanzania. Public Health Nutrition 13(9).

37. Lourme-Ruiz, A., Dury, S., Martin-Prével, Y., 2016. Consomme-t-on ce que l'on sème ? Relations entre diversité de la production, revenu agricole et diversité alimentaire au Burkina Faso. Cah. Agric. 25, 65001. https://doi.org/10.1051/cagri/2016038

38. Macauley, H., Ramadjita, T., 2015. Les cultures céréalières: riz, maïs, millet, sorgho et blé. Groupe de la banque Africaine de Développement. 38p

https://www.afdb.org/fileadmin/uploads/afdb/Documents/Events/Dak Agri2015/Les_cultures_c\%C3\%A9r\%C3\%A9ali\%C3\%A8res_riz 
ma\%C3\%AFs_millet_sorgho_et_bl\%C3\%A9.pdf; consulté le $16 / 06 / 2021$.

39. Maes, K.C., Hadley, C., Tesfaye, F., Shifferaw, S., Tesfaye, Y.A., 2009. Food insecurity among volunteer AIDS caregivers in Addis Ababa, Ethiopia was highly prevalent but buffered from the 2008 food crisis. Journal of Nutrition 139(9), 1758-1764.

40. Malapit, H.J., Kadiyala, S., Quisumbing, A., Cunningham, K., Tyagi, P., 2015. Malapit HJ, Kadiyala S, Quisumbing A, Cunningham K, Tyagi P.Women's empowerment mitigates the negative effects of low production diversity on maternal and child nutrition in Nepal. J Dev Stud 51: 1097-1123. J Dev Stud 51: 1097-1123.

41. Martin-Prével, Y., Allemand, P., Wiesmann, D., Arimond, M., Ballard, T., Deitchler, M., Dop, M.-C., Kennedy, G., Lee, W.T.K., Moursi, M., 2015. Moving forward on choosing a standard operational indicator of women's dietary diversity. Rome: Food and Agriculture Organization of the United Nations. ISBN: 978-92-5-108883-8. 226p http://www.fao.org/3/i4942e/i4942e.pdf (consulté le 17/06/2021).

42. Maxwell, D., Vaitla, B., Coates, J., 2014. Comment les indicateurs d'insécurité alimentaire des ménages se mesurent-ils? Une comparaison empirique de l'Éthiopie. Food Policy 47, 107-116. https://doi.org/10.1016/j.foodpol.2014.04.003

43. Maxwell, S., Frankenberger, T.R., 1992. Household Food Security: Concepts, indicators, Measurements. A Technical Review. New York and Rome : UNICEF and IFAD. 280p. ISBN : 92-806-2021-5.

44. MEPN, M. de l'Environnement et de la P. de la N., 2008. Conventioncadre des nations unies sur les changements climatiques. Fonds pour l'Environnement Mondial (FEM) et le Programme des Nations Unies pour le Développement (PNUD); Appui technique : GIEC, ENDA TM et UNITAR; Cotonou-Republique du Bénin; 81p.

https://unfccc.int/resource/docs/napa/ben01f.pdf (Consulté le 11/10/2020).

45. Michikpe, E., Adégbola, Y.P., 2017. Suivi-Rapproché des Exploitations Familiales Paysannes EFP dans les départements de l'Alibori et du Borgou, au nord-est du Bénin: Analyse de la Sécurité Amentaire et Nutritionnelle (SAN). Document Technique et d'Informations No 04 Rapport fra PNOPPA URP-BAUDOPERBA/INRABILARES/DDC 103 p Dépôt légal No 9273 du 13 mars 2017 Bibliothèque Nationale $(\mathrm{BN})$ du Bénin, 1er trimestre. ISBN : 7899919-2-829-6.

46. Mulumeoderhwa, M.F., Mugisho, M.G., Rushigira, C., Biganiro, M.P., Vwima, N.S., Mushagalusa, N.G., 2020. Strategies d'adaptation 
et securite alimentaire des menages dans les hauts plateaux de minembwe au sud-kivu. Agronomie Africaine 32(2), 207-220.

47. Nagata, J.M., Magerenge, R.O., Young, S.L., Oguta, J.O., Weiser, S.D., Cohen, C.R., 2012. Social determinants, lived experiences, and consequences of household food insecurity among persons living with HIV/AIDS on the shore of Lake Victoria, Kenya. AIDS Care Psychological and Socio-Medical Aspects of AIDS/HIV 24(6), 728736.

48. Ndiaye, M., 2014. INDICATEURS DE LA SÉCURITÉ ALIMENTAIRE.Intégrer les programmes de nutrition et de sécurité alimentaire en situation d'urgence et pour le renforcement de la résilience, Atelier Régional de Formation: 10-12 Juin 2014 Afrique de l’Ouest/Sahel - Saly, Sénégal. 27.

49. Paridaens, A.-M., Ndiayé, A., 2017. République du Bénin: Analyse Globale de la Vulnérabilité et de la Sécurité Alimentaire (AGVSA). Programme Alimentaire Mondial des Nations Unies (PAM), Service de l’Analyse de la Sécurité Alimentaire (VAM) Siège social: Via C.G. Viola 68, Parco de Medici, 00148, Rome, Italie. https://www.insaebj.org/images/docs/insae-statistiques/enquetes-recensements/AutresEnquetes/AGVSA/Rapport_AGVSA_VF_2017.pdf (Consulté le 16/06/2019).

50. PNUD, 2015. Rapport national sur le développement humain (AGRICULTURE, SECURITE ALIMENTAIREET DEVELOPPEMENT HUMAIN AU BENIN).

http://hdr.undp.org/sites/default/files/rndh_2015_benin.pdf (Consulté le 10/01/2020).

51. Pomalegni, S.B.C., Ahoyo Adjovi, N.R., Kpadé, C.P., Gbemavo, D.S.J.C., Allagbé, C.M., Adjanohoun, A., Mensah, G.A., 2019. Capitalisation des études et autres travaux sur les chaînes de valeur du maïs au Bénin. Document Technique et d'Informations (DT\&I). CNSMaïs, INRAB, ProCAD, MAEP, PPAAO/WAAPP, Bénin. Dépôt légal $N^{\circ} 11236$ du 29 avril 2019, 2ème Trimestre, Bibliothèque Nationale (BN) du Bénin. ISBN: 978-99919-75-87-0. 419 p. http://www.slire.net.

52. Prskawetz, A., Winkler-dworak, M., Feichtinger, G., 2003. Prskawetz, A., Winkler-dworak, M., Feichtinger, G.Production, distribution and insecurity of food: a dynamic framework. Structural Change and Economic Dynamics 14, 317-337.

53. Rea, L.M., 1997. Calcul de la taille d'un échantillon pour une enquête, in: Fiches Techniques et Méthodologiques. pp. 713-716. 
54. Regassa, N., Stoecker, B.J., 2012. Household food insecurity and hunger among households in Sidama district, southern Ethiopia. Public Health Nutrition 15(7), 1276-1283.

55. Saliga, F., Alinsato, A., 2021. Analyse de la sécurité alimentaire des ménages agricoles dans le département du Borgou en République du Bénin. Repères et Perspectives Economiques 5(1). https://doi.org/10.34874/IMIST.PRSM/RPE/25756

56. Shariff, Z.M., Khor, G.L., 2005. Obesity and household food insecurity: Evidence from a sample of rural households in Malaysia. European Journal of Clinical Nutrition 59(9), 1049-1058.

57. Sossou, C.H., 2015. Le financement de l'agriculture au Bénin: stratégies de gestion et d'adaptation des exploitations agricoles. Belgique, Université de Liège-Gembloux Agro-Bio Tech. 181 p. Thèse de doctorat (Sciences agronomiques).

58. Temple, L., Jean, M., Moise, K., James, B., Denis, R.D., 2015. Comparaison des trajectoires d'innovation pour la sécurisation alimentaire des pays du Sud. Biotechnol. Agron. Soc. Environ. 19(1), 53-61.

59. Tiyou, A., Belachew, T., Alemseged, F., Biadgilign, S., 2012. Food insecurity and associated factors among HIV-infected individuals receiving highly active antiretroviral therapy in Jimma zone southwest Ethiopia. Nutrition Journal 11(51). DOI:10.1186/1475-2891-11-51.

60. Tsai, A.C., Bangsberg, D.R., Emenyonu, N., Senkungu, J.K., Martin, J.N., Weiser, S.D., 2011. The social context of food insecurity among persons living with HIV/AIDS in rural Uganda. Social Science and Medicine 73(2), 1717-1724.

61. VAM, S. de l'analyse de la sécurité alimentaire, 2014. Approche consolidée du PAM pour le compte-rendu des indicateurs de la sécurité alimentaire (CARI). PAM-Programme Alimentaire Mondial; Via Cesare Giulio Viola, 68/70 — 00148 Rome, Italy. 56p https://docs.wfp.org/api/documents/WFP-0000107746/download/ (Consulté le 17/06/2021).

62. Vellema, W., Deisere, S., D’Haese, M., 2014. Verifying validity of the household dietary diversity score: An application of Rasch Modelling. 2014 International Congress, August 26-29-2014, Slovenia.

63. Yai, E., Yabi, J., Degla, P., Biaou, G., Floquet, A., 2020. Dimon E. Yaï, Jacob A. Yabi, Gauthier Biaou, Anne Floquet, Pamphile Degla, 2020. Productivité agricole et sécurité alimentaires des ménages agricoles du Bénin: Approche des hétérodoxes [ Agricultural productivity and food security of agricultural households in Benin: heterodox approach ]. International Journal of Innovation and Applied Studies 29, 1199-1215. 
64. Yallou, C.G., Aïhou, K., Adjanohoun, A., Baco, M.N., Sanni Ogbon, A., Amadou, L., 2010. Document Technique d'information et de Vulgarisation: Répertoire des Variétés de Maïs (Zea mays L.) Vulgarisées au Bénin. Dépôt légal n 4920 du 3 Décembre 2010, 4ème trimestre. ISBN 978-99919-368-4-0. 19 p.

65. Yegbemey, R.N., Yabi, J.A., Aïhounton, G.B., Paraïso, A., 2014. Modélisation simultanée de la perception et de l'adaptation au changement climatique: cas des producteurs de maïs du Nord Bénin (Afrique de l’Ouest). Cahiers Agricultures 23, 177-187. 